\title{
ПЕРСОНИФИЦИРОВАННАЯ ТЕРАПИЯ КЛИМАКТЕРИЧЕСКОГО СИНДРОМА В СОЧЕТАНИИ С СИНДРОМОМ ПЕРИМЕНОПАУЗАЛЬНОГО ДЕФИЦИТА МЕЛАТОНИНА
}

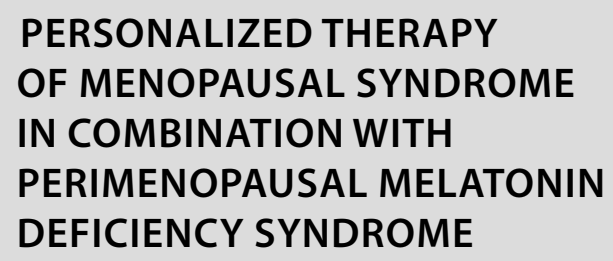

PERSONALIZED THERAPY OF MENOPAUSAL SYNDROME IN COMBINATION WITH

PERIMENOPAUSAL MELATONIN DEFICIENCY SYNDROME

\section{A. Mamontova}

Summary. Menopausal syndrome (MS) is the leading manifestation of pathological menopause. Along with the deficiency of sex steroids in MS, there is a change in the synthesis of the pineal gland hormone melatonin (MT), which leads to the formation of perimenopausal melatonin deficiency syndrome (PMDS). We have developed an algorithm for stratification of women in the menopausal transition and postmenopausal women with MS in combination with PMDS for the selection of differentiated therapy. The purpose of this study was to evaluate the effectiveness of the developed algorithm. The study included 163 women: groups 1, 2, 3, and 4 included patients with MS and sleep disturbance, the control group 5 included women with physiological postmenopause. Patients' quality of life (QOL) was assessed according to a special WHQ questionnaire after collecting their anamnesis, assessing anthropometric data, determining the severity of MS and the hormonal profile. Statistical data processing was carried out using the SPSSv13.0 program. At the initial point of the study before the therapy, the $\chi 2$ criterion was applied and in dynamics - the Wilcoxon test. Based on the study, the high clinical efficacy of the developed algorithm for stratification of women for the selection of differentiated therapy in the menopausal transition and postmenopausal patients with CS in combination with PMDS has been proved.

Keywords: climacteric syndrome, women, melatonin, phytoestrogens, menopausal hormone therapy, quality of life.

\section{Актуальность}

$\mathbf{K}$ лимактерический синдром (КС) является ведущим проявлением патологического климактерия. Наряду с дефицитом половых стероидов при КС наблюдается изменение синтеза гормона эпифиза мелатонина (МТ), что приводит к формированию синдрома перименопаузального дефицита мелатонина (СПДМ) - симптомокомплекса, связанного с нарушением синтеза гормона эпифиза МТ и клинически харак-

\author{
Мамонтова Александра Георгиевна \\ Врач акушер-гинеколог, Соискатель, ГАУЗ ОКБ № 3 \\ (2. Челябинск) \\ Alexandramamontova@rambler.ru
}

Аннотация. Климактерический синдром (КС) является ведущим проявлением патологического климактерия. Наряду с дефицитом половых стероидов при КС наблюдается изменение синтеза гормона эпифиза мелатонина (МТ), что приводит к формированию синдрома перименопаузального дефицита мелатонина (СПДМ). Нами был разработан алгоритм стратификации женщин в менопаузальном переходе и постменопаузе с КС в сочетании с СПДМ для подбора дифференцированной терапии. Целью данного исследования была оценка эффективности разработанного алгоритма. В исследование вошли 163 женщины: в группы 1, 2, 3 и 4 включены пациентки с КС и нарушением сна, в контрольную группу 5 - с физиологически протекающей постменопаузой. После сбора анамнеза, оценки антропометрических данных, определения степени тяжести КС и гормонального профиля пациенток оценивалось их качество жизни (КЖ) по данным специального опросника WHQ. Статистическая обработка данных осуществлялась с помощью программы SPSSv13.0. В начальной точке исследования до старта терапии применен критерий Х2 и в динамике - тест Вилкоксона. На основании проведенного исследования доказана высокая клиническая эффективность разработанного алгоритма стратификации женщин для подбора дифференцированной терапии в менопаузальном переходе и постменопаузе при КС в сочетании с СПДМ.

Ключевые слова: климактерический синдром, женщины, мелатонин, фитоэстрогены, менопаузальная гормональная терапия, качество жизни.

теризующийся доминированием жалоб на нарушение сна, болевых ощущений в теле, наличием депрессии, тревоги/страхов и соматических симптомов (усталость, головокружение, тошнота; боль в спине, руках/ногах; покалывание в кистях рук/ступнях) [1]. КС и СПДМ являются коморбидными состояниями, нарушающими климактерическое здоровье женщин [2], а подходы к дифференцированному лечению данной патологии требуют дальнейшего изучения, что и определило цель нашего исследования. 


\section{Шель исслеАования}

Цель данного исследования заключалась в определении эффективности дифференцированного подхода в лечении женщин с КС в сочетании с СПДМ.

\section{Материалы и метолы}

В исследование вошли 163 женщины. В группы 1, 2, 3 и 4 включены пациентки, которые предъявляли жалобы на наличие климактерических симптомов и нарушение сна. В контрольную группу 5 вошли женщины с физиологически протекающей постменопаузой.

Пациентки, обратившиеся на прием по проблемам климактерия, были разделены на группы, согласно разработанному нами алгоритму стратификации женщин для дифференцированной терапии КС в сочетании с СПДМ [3].

В группу 1 (n=43) были включены женщины с КС легкой степени и СПДМ, в том числе при доминировании жалоб на нарушение сна, наличие депрессии, повышенной утомляемости, а также больные с КС любой степени и СПДМ при наличии противопоказаний к фитоэстрогенам (ФЭ) и менопаузальной гормональной терапии (МГТ). Этим пациенткам назначена монотерапия мелатонином (МТ) по 3 мг за 30 мин до сна на протяжении 3 месяцев. В группу $2(\mathrm{n}=41)$ включены пациентки с КС легкой степени и СПДМ при наличии противопоказаний к назначению МТ, а также женщины с КС любой степени тяжести и СПДМ, которые отказались от МТ и МГТ. Данным пациенткам назначен ФЭ- синтетический гинестеин по 30 мг 2 раза в день в течение 3 месяцев. Группа $3(\mathrm{n}=24)$ состояла из женщин с КС средней и тяжелой степени в сочетании с СПДМ, которые получали комбинированную терапию - препараты МГТ и МТ не менее 3 месяцев. В группу $4(n=25)$ включены женщины с KC различной степени тяжести и СПДМ при наличии противопоказаний к назначению МГТ. Пациенткам назначена комбинированная терапия - МТ и синтетический гинестеин (ФЭ) в течение 3 месяцев. Контрольная группа $5(n=30)$ находилась под динамическим наблюдением врача. Всем пациенткам проводился сбор анамнеза, определение модифицированного менопаузального индекса (ММИ) (Kupperman H. et al. 1959 г, в модификации Уваровой Е.В. 1983 г.) [4], индекса массы тела (ИМТ), соотношение окружности талии к окружности бедер (ОТ/ОБ). Для выявления дефицита МТ использовался метод анкетирования, применялся тест-опросник на дефицит МТ (ТОДМ) [5], при этом показатели от 0 до 4 баллов соответствовали норме, результат от 5 до 9 баллов свидетельствовал о том, что уровень МТ начинал снижаться, а от 10 до 14 баллов регистрировался явный дефицит MT [5]. С помощью лабораторного метода (ИФА) прово- дилось определение концентрации ФСГ, ЛГ, эстрадиола, общего тестостерона и пролактина сыворотки крови.

Для оценки параметров качества жизни (КЖ) пациентки заполняли специальный опросник WHQ (Women's Health Questionnaire) (автор — dr. MyraHunter, Department of Psychology Adamson Centre ST Thomas Hospital Lambeth Palace Road, London, UK) [6, 7], психометрические свойства которого в среде русскоязычной популяции изучены отечественными авторами [8, 9]. Опросник состоит из 37 вопросов, которые объединяются в 9 шкал $[6,7,8]$ : «депрессия» (Д), «физические/соматические симптомы» (ССМ), «память» (П), «вазомоторные симптомы» (ВСМ), «тревожность/страх» (ТС), «сексуальное нарушения» (CH), «проблемы со сном» (C), «менструальные симптомы», «привлекательность» (П). Каждая шкала имеет размерность от 0 до 1 балла, чем ближе значение к 0, тем лучше КЖ респондента. Оценка вопросов проводится по четырехбалльной системе [8]. Шкала «менструальные симптомы» нами не анализировалась ввиду того, что часть пациенток имела длительную аменорею. [8].

Эффективность разработанного алгоритма стратификации женщин для дифференцированной терапии КС в сочетании с СПДМ была оценена в четырех группах в нескольких точках исследования (через 1 и 2-3 месяца и через 5-6 месяцев). Статистическая обработка данных производилась с помощью программы SPSSv.13.0. По критерию Вилкоксона в динамике оценивались изменения в группах веса, индекса массы тела (ИМТ), соотношения окружности талии и бедер (ОТ/ОБ), модифицированного менопаузального индекса (ММИ), результата теста-опросника на дефицит МT.

\section{Результаты}

На первом этапе был проведен сравнительный анализ между всеми группами до старта терапии. Средний возраст пациенток не имел статистически значимых отличий и в группе 1 составил 53,2 6,9 года, в группе $2-52,8 \pm 6,2$ лет, в группе $3-51,7 \pm 4,5$ лет, в группе $4-53,9 \pm 4,6$ лет и в группе $5-52,2 \pm 5,3$ года $(x 2=0,04$; $\mathrm{p} 1-5=0,85)$.

При анализе антропометрических данных ИМТ в группах 1, 2 и 3 соответствовал избыточной массе тела $(27,6 \pm 4,7 ; 27,3 \pm 5,3$ и 26,5 \pm 5 , соответственно), а в груп-

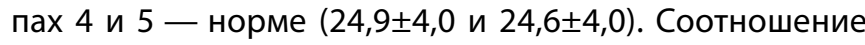
ОТ/ОБ было менее 0,85 во всех группах, что соответствовало нормальному распределению жировой ткани. Статистически значимых различий по антропометрическим данным между группами выявлено не было.

Статистически значимо группы отличались по ММИ, при этом у пациенток групп 1-4 он соответствовал 
Таблица 1. Показатели модифицированного менопаузального индекса обследованных женщин в начальной точке исследования

\begin{tabular}{|c|c|c|c|c|c|c|c|}
\hline \multirow[t]{2}{*}{ Показатели } & Группа 1 n=43 & $\begin{array}{l}\text { Группа } 2 \\
\mathrm{n}=41\end{array}$ & $\begin{array}{l}\text { Группа } 3 \\
\mathbf{n}=24\end{array}$ & $\begin{array}{l}\text { Группа } 4 \\
n=25\end{array}$ & $\begin{array}{l}\text { Группа } 5 \\
\mathrm{n}=30\end{array}$ & \multirow[t]{2}{*}{ Хи-квадрат } & \multirow{2}{*}{$\begin{array}{l}\text { Уровень } \\
\text { значимости р }\end{array}$} \\
\hline & Me (LQ; UQ) & Me (LQ; UQ) & Me (LQ; UQ) & Me (LQ; UQ) & Me (LQ; UQ) & & \\
\hline ММИ, баллы & $\begin{array}{l}26 \\
(20 ; 32)\end{array}$ & $\begin{array}{l}22 \\
(15,5 ; 28)\end{array}$ & $\begin{array}{l}26,5 \\
(22 ; 32,7)\end{array}$ & $\begin{array}{l}21 \\
(15 ; 27,5)\end{array}$ & $\begin{array}{l}7,5 \\
(6 ; 9)\end{array}$ & 6 & 0,01 \\
\hline
\end{tabular}

Примечание: ММИ-модифицированный менопаузальный индекс

Таблица 2. Показатели уровня качества жизни групп в начальной точке исследования по данным специального опросника WHQ

\begin{tabular}{|c|c|c|c|c|c|c|c|}
\hline $\begin{array}{l}\text { Шкалы WHQ/ } \\
\text { Точки иссле- } \\
\text { дования }\end{array}$ & $\begin{array}{l}\text { Группа } 1 \\
\text { n=43 Me (LQ; } \\
\text { UQ) }\end{array}$ & $\begin{array}{l}\text { Группа } 2 \\
n=41 \text { Me (LQ; } \\
\text { UQ) }\end{array}$ & $\begin{array}{l}\text { Группа } 3 \\
\text { n= } 24 \text { Me (LQ; } \\
\text { UQ) }\end{array}$ & $\begin{array}{l}\text { Группа } 4 \\
\text { n=25 Me (LQ; } \\
\text { UQ) }\end{array}$ & $\begin{array}{l}\text { Группа } 5 \\
n=30 \text { Me (LQ; } \\
\text { UQ) }\end{array}$ & Хи-квадрат & $\begin{array}{l}\text { Уровень } \\
\text { значимости F }\end{array}$ \\
\hline \multicolumn{8}{|l|}{ T0 } \\
\hline Д & $\begin{array}{l}0,43(0,29 ; \\
0,57)\end{array}$ & $\begin{array}{l}0,29(0,14 ; \\
0,42)\end{array}$ & $\begin{array}{l}0,43(0,29 ; \\
0,57)\end{array}$ & $\begin{array}{l}0,29(0,14 ; \\
0,43)\end{array}$ & $\begin{array}{l}0,29(0,14 ; \\
0,43)\end{array}$ & 4,19 & 0,04 \\
\hline CCM & $\begin{array}{l}0,57(0,43 ; \\
0,86)\end{array}$ & $\begin{array}{l}0,71(0,29 ; \\
0,86)\end{array}$ & $\begin{array}{l}0,71 \\
(0,57 \\
0,86)\end{array}$ & $\begin{array}{l}0,43(0,29 \\
0,86)\end{array}$ & $\begin{array}{l}0,29(0,14 ; \\
0,43)\end{array}$ & 0,16 & 0,69 \\
\hline$\Pi B$ & $\begin{array}{l}0,67(0,33 ; \\
1,0)\end{array}$ & $0,67(0 ; 1)$ & $0,67(0,33 ; 1)$ & $0,67(0,33 ; 1)$ & $0,33(0 ; 0,67)$ & 0,74 & 0,39 \\
\hline $\mathrm{BCM}$ & $\begin{array}{l}1,0(0,5 ; \\
1,0)\end{array}$ & $1(0,5 ; 1)$ & $1(1 ; 1)$ & $1(0,5 ; 1)$ & $0,5(0,5 ; 1)$ & 1,13 & 0,29 \\
\hline TC & $\begin{array}{l}0,50(0,25 ; \\
0,75)\end{array}$ & $0,25(0 ; 0,5)$ & $0,50(0,25 ; 0,75)$ & $0,25(0 ; 0,63)$ & $0,25(0 ; 0,5)$ & 5,23 & 0,02 \\
\hline C & $0,67(0,33 ; 1)$ & $0,67(0,33 ; 0,67)$ & $0,67(0,33 ; 0,67)$ & $0,67(0,33 ; 0,67)$ & $0,33(0,33 ; 0,67)$ & 0,19 & 0,05 \\
\hline $\mathrm{CH}$ & $\begin{array}{l}0,67(0,33 ; \\
1,0)\end{array}$ & $0,67(0 ; 0,67)$ & $0,5(0,33 ; 1)$ & $0,67(0,33 ; 1)$ & $0,33(0 ; 0,67)$ & 3,83 & 0,05 \\
\hline$\Pi$ & $0(0 ; 1,0)$ & $0,5(0 ; 1)$ & $0(0: 0,5)$ & $0,5(0 ; 1)$ & $0,5(0 ; 1)$ & 3,88 & 0,05 \\
\hline
\end{tabular}

Примечания: Д-депрессия, ССМ-соматические симптомы, ПВ-память/внимание, ВСМ-вазомоторные симптомы, ТС-тревога/страхи, С-сон, СН-сексуальные нарушения, П-привлекательность

легкой степени КС, а у пациенток группы 5 - ММИ был в норме $(p=0,01)$ (таб. 1).

Статистически значимые различия были получены при сравнении показателей ТОДМ. Данный показатель в группе 1-8 $(6 ; 10)$ и 3-8 $(7 ; 11)$ баллов, в группе 4-7 (5; 8) и в группе $2-6(4 ; 8)$ баллов, что свидетельствовало о снижении МТ у пациенток. В группе 5 медиана данного показателя составила $4,5(3 ; 5,8)$ балла, что является пограничным значением между нормальным и сниженным уровнем гормона эпифиза $(p=0,001)$.

Статистически значимых различий между группами по уровню гормонов сыворотки крови не было выявлено.
Таким образом, статистически значимые различия между группами в начальной точке исследования наблюдались по показателям ММИ, по результатам теста-опросника на дефицит МТ, что являлось ожидаемым результатом ввиду применения нами алгоритма стратификации женщин для дифференцированной терапии КС в сочетании с СПДМ.

Для оценки уровня КЖ пациенткам предлагалось заполнить опросник WHQ. В Т0 при анализе шкалы Д были выявлены статистически значимые различия между группами, при этом наиболее низкое КЖ по данной шкале наблюдалось в группе 1 и $3(p=0,04)$. Остальные группы были сопоставимы по данному показателю, где его медиана составила 0,29 балла. По шкале ТС группы 


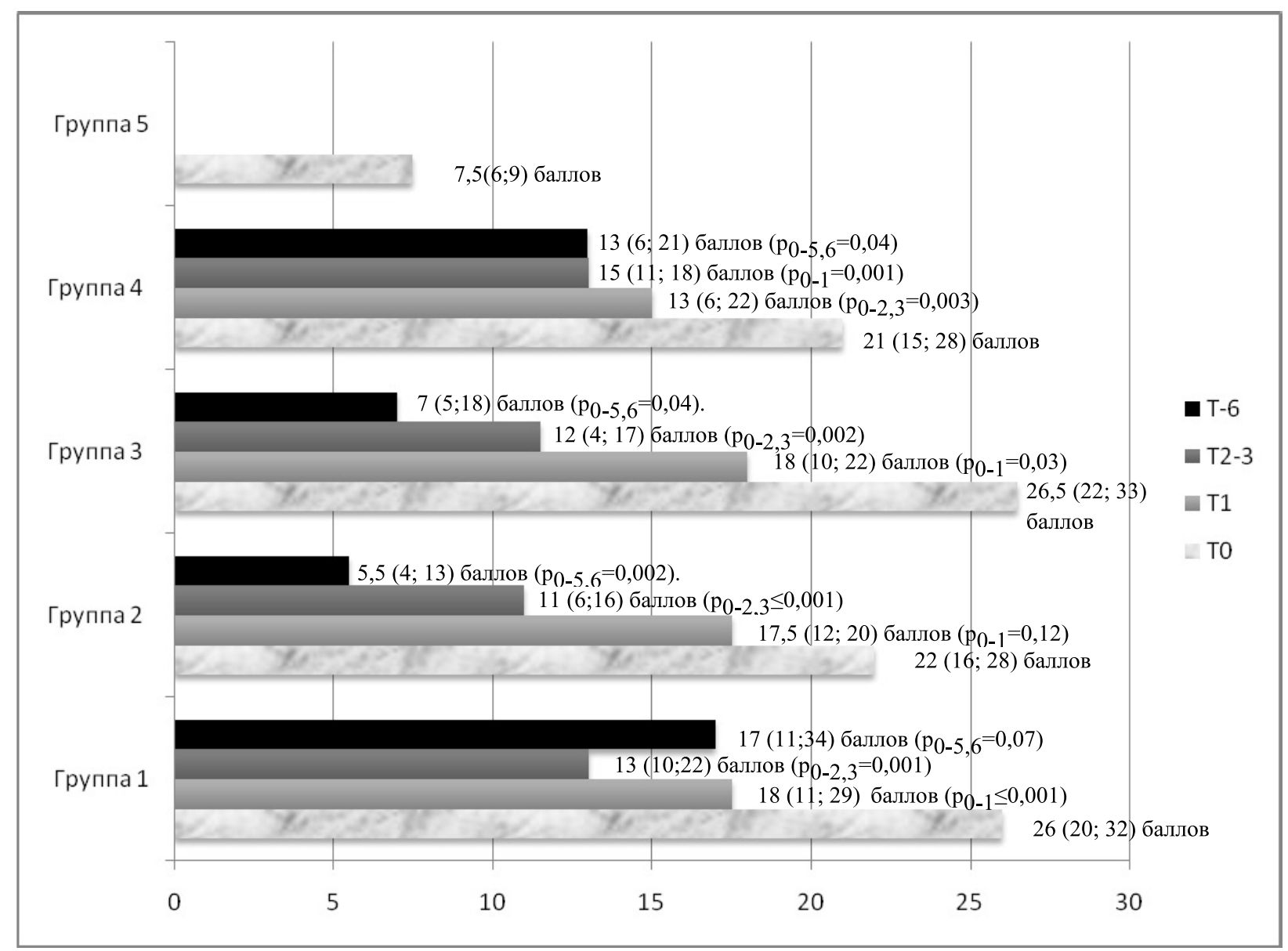

Рис. 1. Динамика показателей модифицированного менопаузального индекса при стратификации женщин для лечения климактерического синдрома в сочетании с синдромом перименопаузального дефицита мелатонина

также отличались, при этом в группе 1 и 3 Кж было низким, а в группах 2, 4, 5 регистрировался достаточно высокий уровень КЖ ( $p=0,02)$. Проблемы со сном имелись в группах 1, 2, 3, 4, а в контрольной группе шкала С была в норме $(p=0,05)$. По данным остальных шкал опросника WHQ статистически значимых различий не обнаружено (таб. 2).

Таким образом, статистически значимые различия между группами наблюдались по шкалам Д, ТС, С, что является одним из диагностических критериев СПДМ у пациенток с КС.

На втором этапе исследования была оценена эффективность лечения с позиций динамики клинических симптомов и параметров КЖ.

Антропометрические данные. При анализе антропометрических данных (ИМТ и соотношение ОТ/ОБ) на фоне лечения не выявлено статистически значимых изменений ни в одной из групп.
Модифицированный менопаузальный индекс (ММИ). В процессе лечения было зарегистрировано снижение значений ММИ во всех группах (рис. 1). В группе 1 ММИ начал снижаться через 1 месяц лечения, положительная динамика сохранилась к 2-3 месяцам терапии, а через 5-6 месяцев лечения было отмечено незначительное увеличение ММИ, что может быть связано с небольшим количеством наблюдений в финальной точке. $(\mathrm{n}=4)$. В группе 2 также наблюдалось снижение показателей ММИ уже через 1 месяц терапии. Данный показатель продолжил снижаться через 2-3 месяца терапии, а через 5-6 месяцев лечения достиг нормы. В группе 3 через 1 месяц лечения ММИ начал снижаться. Положительная динамика сохранялась через 2-3 месяцам лечения, а через 5-6 месяцев лечения данный показатель достиг нормальных значений. В группе 4 снижение ММИ произошло также через 1 месяц терапии. Степень тяжести КС продолжала снижаться, через 2-3 месяца терапии и сохранилась практически на том же уровне через 5-6 месяцев. 
Таблица 3. Сравнительный анализ качества жизни групп по критерию Х2 (WHQ)

\begin{tabular}{|c|c|c|c|c|c|c|}
\hline \multirow{2}{*}{ Шкалы WHQ } & \multicolumn{2}{|l|}{ T1 } & \multicolumn{2}{|c|}{ T2-3 } & \multicolumn{2}{|l|}{ T5-6 } \\
\hline & $x^{2}$ & $p$ & $x^{2}$ & $p$ & $x^{2}$ & $p$ \\
\hline Д & 7,96 & 0,05 & 1,75 & 0,63 & 7,86 & 0,05 \\
\hline CCM & 3,02 & 0,39 & 2,63 & 0,45 & 2,83 & 0,42 \\
\hline$\Pi \mathrm{B}$ & 2,68 & 0,44 & 1,94 & 0,59 & 1,70 & 0,64 \\
\hline $\mathrm{BCM}$ & 1,86 & 0,60 & 5,00 & 0,17 & 0,91 & 0,82 \\
\hline TC & 4,03 & 0,26 & 1,82 & 0,61 & 9,76 & 0,02 \\
\hline $\mathrm{CH}$ & 1,88 & 0,60 & 1,79 & 0,62 & 5,31 & 0,15 \\
\hline C & 3,21 & 0,36 & 1,66 & 0,65 & 2,32 & 0,51 \\
\hline$\Pi$ & 8,01 & 0,05 & 1,04 & 0,79 & 5,20 & 0,16 \\
\hline
\end{tabular}

Примечание: Д-депрессия, ССМ-соматические симптомы, ПВ-память/внимание, ВСМ-вазомоторные симптомы, ТС-тревога/страхи, С-сон, СН-сексуальные нарушения, П-привлекательность

Результаты теста-опросника на дефицит мелатонина. В группе 1 показатели ТОДМ начали снижаться уже через 1 месяц от начала терапии, достигнув $4(2 ; 9)$ баллов $\left(p_{0-1}=0,01\right)$, через 2-3 месяца - 5 (33; 7) баллов $\left(p_{0-2,3} \leq 0,001\right)$, а через 5-6 месяцев лечения ТОДМ - $4(3,3 ; 6,3)$ балла.

В группе 2 не было выявлено статистически значимых изменений показателей ТОДМ.

В группе 3 через 1 месяц лечения баллы ТОДМ снизились до 5 (4; 11), но статистически значимых изменений не было выявлено, через 2-3 месяца терапии данный показатель достиг $3(2 ; 6)$ баллов $\left(p_{0-2,3} \leq 0,001\right)$. Через 5-6 месяцев лечения результаты ТОДМ составили $5(1 ; 8)$ баллов.

В группе 4 в точке Т1 баллы по ТОДМ снизились до 4 $(2 ; 6)\left(p_{0-1}=0,01\right)$. Через 2-3 месяц лечения показатели ТОДМ продолжили снижаться и достигли $2(1 ; 5)$ баллов (р0-2,3=0,05), а в финальной точке исследования данный показатель составил $4(4 ; 5)$ балла $\left(p_{0-5,6}=0,04\right)$.

В ходе данного исследования был проведен анализ клинической эффективности персонифицированного лечения женщин с КС в рамках межгруппового анализа. Проведено сравнение групп по критерию $\mathrm{X}^{2}$ в точке Т1 (через 1 месяц терапии), в точке Т2-3 (через 2-3 месяца терапии) и в Т5-6 (через 5-6 месяцев лечения) по данным ММИ и его компонентам, а также по результатам ТОДМ. Статистически значимых различий между группами не было выявлено ни в одной точке.

Таким образом, разработанный нами алгоритм стратификации женщин для дифференцированной терапии КС в сочетании с СПДМ у женщин в периоде менопаузального перехода и постменопаузы оказался эффективным во всех четырех группах. Так, значения ММИ в группах 1, 2, 3 и 4 значительно снизились уже через 1 месяц терапии, положительный эффект нарастал к 2-3 месяцам и достиг максимального значения к 5-6 месяцам лечения. При анализе компонентов ММИ установлено, что нейровегетативные, обменно-эндокринные и психоэмоциональные симптомы максимально эффективно купировали ФЭ и комбинированная терапия - МГТ в сочетании с МТ. Антропометрические показатели остались без изменений на фоне дифференцированного лечения. Качество сна по данным ТОДМ улучшилось в большей степени под влиянием МТ при монотерапии, а также при комбинированной терапии МГТ в сочетании с МТ или ФЭ с МТ. Положительного влияния на качество сна препаратов ФЭ не установлено.

Динамика показателей КЖ на фоне лечения. Эффективность разработанного алгоритма стратификации женщин для лечения КС в сочетании с СПДМ оценена в четырех группах в нескольких точках исследования с помощью анализа динамики показателей КЖ пациенток как в каждой группе отдельно, так и между группами.

В группе 1 через 1 месяц терапии наблюдалось улучшение КЖ по шкале ТС $\left(p_{0-1}=0,05\right)$, а к 2-3 месяцам - статистически значимое улучшение КЖ по ряду шкал: Д $\left(p_{0-2,3}=0,01\right)$, CC $\left(p_{0-2,3} \leq 0,01\right)$, TC $\left(p_{0-2,3}=0,05\right)$ и $C\left(p_{0-2,3}=0,01\right)$ (таб. 3).

В группе 2 через 1 месяц не зарегистрировано значительное улучшение КЖ. Через 2-3 месяца лечения положительный статистически значимый эффект от лечения был установлен по таким шкалам как: Д $\left(\mathrm{p}_{0-2,3}=0,01\right), \mathrm{CC}\left(\mathrm{p}_{0-2,3} \leq 0,01\right), \mathrm{TC}\left(\mathrm{p}_{0-2,3}=0,05\right)$. Положительное, но статистически незначимое влияние было зарегистрировано по шкалам $B C\left(p_{0-2,3}=0,07\right)$ и $\Pi\left(p_{0-2,3}=0,06\right)$. К 5-6 мес. терапии значительно улучшилось КЖ по шкале $B C\left(p_{0-5,6}=0,04\right)$. По шкале П наблюдалось улучшение КЖ, но статистически незначимое $\left(p_{0-5,6}=0,07\right)$. 
В группе 3 выявлены тенденции улучшения КЖ на фоне терапии через 1 месяц лечения, однако статистически незначимо, а через 2-3 месяца было зарегистрировано по шкале ВC $\left(\mathrm{p}_{0-2,3}=0,05\right), \mathrm{CC}\left(\mathrm{p}_{0-2,3}=0,06\right)$ и ТС $\left(\mathrm{p}_{0-2,3}=0,06\right)$. К 5-6 месяцам лечения значительно улучшилось КЖ по шкале ТС $\left(p_{0-5,6}=0,03\right)$.

В группе 4 улучшение КЖ было зарегистрировано через 2-3 месяца лечения, при этом наиболее значимо уровень КЖ увеличился по таким шкалам как СС $\left(p_{0-2,3}=0,04\right), \Pi B\left(p_{0-2,3}=0,04\right)$ и $B C\left(p_{0-2,3}=0,04\right)$.

Согласно полученным данным через 1 месяц лечения статистически значимые различия между группами выявлены по шкалам Д ( $\left.p_{0-1}=0,05\right)$ и П $\left(p_{0-1}=0,05\right)$. При этом по шкале Д самое низкое КЖ наблюдалась в группе 1 и 3, а в группах 2 и 4 было высоким. По шкале П - КЖ было низким в группах 2 и 4 и высоким в группе 1 и 3. Через 2-3 месяца терапии все группы были сопоставимы по КЖ и статистически значимо не различались друг от друга. Через 5-6 месяцев лечения группы отличались только по шкале Д $\left(p_{0-5,6}=0,05\right)$, при этом высокое КЖ было зарегистрировано в группе 1, во всех остальных КЖ было по данной шкале было одинаково низким.

При анализе изменений уровня КЖ согласно данным специального опросника WHQ монотерапия МТ в большей степени улучшила показатели шкал Д, СС, ТС и С, в то время как монотерапия ФЭ дополнительно положительно влияла на ВС, но не улучшала сон (C) женщин. Комбинированная терапия МТ и препаратами МГТ в большей степени оказывала влияние на шкалы ВС и ТС, а комбинированная терапия МТ и ФЭ улучшила показатели ВС, СС и ПВ.

\section{Выво $\Delta$}

В результате проведенного исследования установлено, что наряду с клинической эффективностью предложенных подходов в лечении КС и СПДМ выявлена положительная динамика показателей КЖ женщин, что подчеркивает научную обоснованность и практическую значимость разработанного алгоритма стратификации женщин для дифференцированной терапии КС в сочетании с СПДМ в периоде менопаузального перехода и постменопаузе.

\section{ЛИТЕРАТУРА}

1. Усольцева, Е.Н. Синдром перименопаузального дефицита мелатонина в системе персонифицированного менеджмента качества жизни женщин с патологическим климактерием / Е.Н. Усольцева, А.Г. Соловьев, А.Г. Мамонтова // Успехи геронтологии. — 2019. — Т. 32. — № 4. — С. $516-523$.

2. Усольцева, Е.Н. Качество жизни и пути его улучшения у женщин с климактерическими расстройствами: автореферат дис. ... д-ра мед. наук / Е.Н. Усольцева. — Челябинск: Южно-Уральский государственный медицинский университет, 2018.

3. Мамонтова, А.Г. Алгоритм стратификации женщин для дифференцированной терапии климактерического синдрома в сочетании с синдромом перименопаузального дефицита мелатонина / А.Г. Мамонтова, Е.Н. Усольцева, А.Г. Соловьев // Успехи геронтологии. — 2020. — Т. 33. — № 6. — C. 1137-1141.

4. Сметник, В.П. Медицина климактерия / В.П. Сметник. — Ярославль: Литера. 2006. - 848 с.

5. Dalle C. The Guide. Global Aging Management. [S.I.: s.n]. — 2010. — № 1. —:496 p.

6. Kanadys, K. Predictors of the quality of life of women in peri-menopausal period / K. Kanadys, A. Wiktor-Stoma, M. Lewicka, M. Sulima, H. Wiktor //Annals of Agricultural and Environmental Medicine. — 2016. — 23(4). — P. 641-648.

7. Katainen, Riina Climacteric-related symptoms in midlife and beyond - studies using the women's health questionnaire / Riina Katainen. // Turun Yliopiston Julkaisuja — Annales Universitatis Turkuensis Sarja — Ser. D 0sa. — T. 1394. — Medica — Odontologica | Turku. — 2018.

8. Брюхина, Е.В. Психометрические свойства русскоязычной версии опросника женского здоровья women's health questionnaire (WHQ) / Е.B. Брюхина, О.В. Иванова, Е.Н. Усольцева // Вестник Южно-Уральского государственного университета. Серия: Образование, здравоохранение, физическая культура. — 2012. — № 42 (301). — C. 134-142. — Режим доступа: URL: https://cyberleninka.ru/article/n/psihometricheskie-svoystva-russkoyazychnoyversii-oprosnika-zhenskogo-zdorovya-women-s-health-questionnaire-whq

9. Иванова, 0.В. Валидация русскоязычной версии опросника “Women's health questionnaire (WHQ)" / О.В. Иванова, Е.Н. Усольцева, Е.В. Брюхина // Вестник межнационального центра исследования качества жизни. -2011.— № 17-18. — C. 58-68.

(с) Мамонтова Александра Георгиевна ( Alexandramamontova@rambler.ru ).

Журнал «Современная наука: актуальные проблемы теории и практики» 